\title{
Baukastensystem für CFK-Fachwerkstrukturen - Diskrete Optimierung der Strebenquerschnitte
}

Simon Walbrun ${ }^{1}$, Sandro Wartzack ${ }^{1}$

${ }^{1}$ Engineering Design (KTmfk)

Friedrich-Alexander-Universität Erlangen-Nürnberg (FAU), Germany

\section{Abstract}

Fiber-reinforced plastics have a high potential for lightweight structures due to their high specific strength and stiffness. A modular system enables a reduction of the manufacturing costs for trusses by standardizing the components. The subdivision of the truss struts into groups and the selection of the profiles for the strut groups can be done by optimization with a genetic algorithm and the initial population generation by the bisection method. The proposed method reduces the number of necessary iterations and improves the optimum in comparison to the previous descriptions.

Keywords: Genetic algorithms, Initial population, Optimization, Self-adaptive member grouping

\section{Hybrider Leichtbau für Faserverbundfachwerke}

Faserverstärkte Kunststoffe (FVK) haben durch ihre hohe richtungsabhängige spezifische Festigkeit und Steifigkeit Vorteile im Vergleich zu herkömmlichen metallischen Werkstoffen, insofern die Anisotropie des Materials in der Konstruktion ausgenutzt wird [1]. Beim hybriden Leichtbau werden mehrere Materialien entsprechend ihrer mechanischen Eigenschaften und der auftretenden Beanspruchungen in einem Produkt kombiniert. Bei Fachwerken treten in 
den Streben einachsige Spannungszustände und in den Verbindungselementen mehrachsige Spannungszustände auf, wie von KLEIN [2] an einem Demonstrator gezeigt. Da das Potential von FVK bei einem einachsigen Spannungszustand am besten genutzt werden kann und Metalle auch mehrachsige Spannungszustände ertragen können, ist FVK für die Streben und Metall für die Verbindungselemente prädestiniert. Bei hybriden Fachwerken kann im Vergleich zu rein metallischen Konstruktionen bei gleicher Masse eine Erhöhung der Steifigkeit um $20 \%$ erwartet werden [3]. Zudem kann bei einer Struktur die gleiche Steifigkeit bei geringerer Masse verwirklicht werden, wodurch sich bei bewegten und beschleunigten Bauteilen deutliche Energieeinsparrungen erzielen lassen. Die hohe Schwingungsdämpfung in Kombination mit der erhöhten Steifigkeit führt zusätzlich zu einer Erhöhung der Eigenfrequenzen bei gleichzeitiger Reduktion der Schwingungsamplituden [1].

Neben vereinzelten Anwendungen von FVK-Fachwerken im Maschinenbau zur Nutzung der hohen Steifigkeit, ist derzeit der Einsatz weitestgehend auf die Sektoren Luft- und Raumfahrt beschränkt, da die hohen Produktentwicklungsund Fertigungskosten durch reduzierte Kosten während des Betriebs ausgeglichen werden. Die fehlende Standardisierung der Fertigung und Produktion verursacht zusätzliche Kosten, welche zu den vergleichsweise hohen Werkstoffkosten hinzukommen. Ein Bauteil, welches aus kohlenstofffaserverstärktem Kunststoff gefertigt wird, verursacht im Vergleich zur Ausführung in Stahl in etwa die siebenfachen Kosten, wobei $50 \%$ auf den Prozess und $50 \%$ auf das Material entfallen [4]. Die derzeitigen Fertigungs- und Entwicklungsverfahren von FVK sind nicht geeignet für die effiziente Produktion von beliebigen räumlichen Fachwerkstrukturen. Die Entwicklung einer Konstruktionsmethode welche, von der Topologieoptimierung bis zur CAD-Modell Erzeugung, eine CAE Unterstützung gewährleistet ermöglicht die Verwendung von FVK-Fachwerken in weiteren Anwendungen. Basis des Systems soll hierbei ein Baukasten mit standardisierten Profilen und modularen Verbindungselementen sein. Durch die damit einhergehende Standardisierung und Automatisierung können die Prozesskosten gesenkt werden.

Die Auswahl der Strebenquerschnitte ist ein wesentlicher Auslegungsschritt bei gegebener Skelettstruktur des Fachwerks, wobei die Masse minimiert und die Steifigkeit des Fachwerks einen gesetzten Sollwert erreichen soll. Dies gestaltet sich als Optimierungsaufgabe mit diskreten Parametern. Als standardisierte Streben werden gewickelte runde Hohlprofile verschiedener Wandstärken und Durchmesser verwendet. 
Die numerische Optimierung von Fachwerken mit kontinuierlichen Variablen wurde von DORN [5] eingeführt. Die Querschnittsflächen der Streben werden als Variable optimiert mit dem Ziel die Masse zu minimieren. Die erste Optimierung mit diskreten Variablen wurde von TOAKLEY [6] für statisch bestimmte Fachwerke durchgeführt. Wie von STOLPE [7] beschrieben kann die Optimierungsaufgabe mit Meta-Heuristischen, Deterministischen und Heuristischen Verfahren gelöst werden. Die Meta-Heuristischen Verfahren und insbesondere der Genetische Algorithmus (GA) haben sich hierbei als besonders effizient und geeignet gezeigt, da aufgrund der diskreten Variablen keine Gradienten gebildet werden können.

Die Formulierung des Minimierungsproblems mit den Nebenbedingungen ist [7]:

$$
\begin{aligned}
& \min (m(\boldsymbol{x}))=\sum_{i=1}^{I} A_{i} l_{i} \rho_{i} \\
& u_{\min } \leq u_{i}(\boldsymbol{x}) \leq u_{\max } \\
& \sigma_{\min }(\boldsymbol{x}) \leq \sigma_{i}(\boldsymbol{x}) \leq \sigma_{\max }(\boldsymbol{x}) \\
& x_{i} \in S=\left\{s_{1}, s_{2}, \ldots, s_{M}\right\}
\end{aligned}
$$

Hierbei ist $m$ die Masse des Fachwerks, in Abhängigkeit der Strebenprofile $x$ und ergibt sich aus der Querschnittsfläche $A$, der Länge $l$ und der Dichte $\rho$. Die Verschiebungen $u$ der Knoten und die Spannungen $\sigma$ müssen zwischen den entsprechenden Grenzwerten liegen. $S$ ist die Menge an möglichen Profilen der Anzahl $M$.

Die Lösung des Optimierungsproblems wurde u.a. von RAJEeV [8-10] beschrieben und für mehrere Fachwerke gelöst. Es wurde gezeigt, dass genetische Algorithmen geeignet sind die Optimierungsaufgabe zu lösen. Um die Anzahl an Freiheitsgraden zu reduzieren, werden die Streben im Vorfeld der Optimierung manuell in mehrere Gruppen mit gleichen Querschnitten unterteil. Der GA startet mit meist zufällig ausgewählten potentiellen Lösungen und entwickelt diese iterativ durch die Operatoren Selektion, Vererbung und Mutation weiter. Durch die Auswertung der Zielfunktion und Nebenbedingungen werden die fittesten Individuen ausgewählt. Es ist keine Auswertung von Gradienten nötig wodurch auch unstetige Funktionen optimiert werden können [11].

Von Togan $[10,12]$ wurde die Gruppierung der Streben und die Erzeugung einer Anfangspopulation durch eine vorgelagerte Berechnung vorgeschlagen. 
Die Gruppierung der Streben erfolgt in Abhängigkeit der vorhandenen axialen Beanspruchungen und wird während der Optimierung beibehalten. Die Anfangspopulation wird von TOGAN [10] über die Festigkeit bestimmt. Diese Annahmen vernachlässigen, dass die Nebenbedingung der maximalen Verschiebung oft limitierender Faktor bei der Auslegung ist. Somit stellt die Einteilung der Streben in fixe Gruppen eine Limitierung dar, welche in dieser Arbeit betrachtet wird. Die Erzeugung einer entsprechenden Anfangspopulation für den GA wird ebenfalls vorgestellt.

\section{Methode zur diskreten Optimierung}

In dieser Arbeit wird ein Ansatz präsentiert für die automatische Einteilung der Streben in die Gruppen und das Erstellen einer Anfangspopulation für den GA. Die Anzahl an Strebengruppen ist eine frei wählbare Nebenbedingung. Die Anfangspopulation wird mithilfe des Intervallhalbierungsverfahrens [11] ermittelt.

\subsection{Bauteilgruppenbildung}

Bei einer gegebenen Struktur können die Querschnitte sämtlicher Streben als Freiheitsgrad verwendet werden. Dies hat den Nachteil, dass die Rechenzeit hoch ist und die Gefahr besteht, dass ein lokales Minimum gefunden wird [10]. Durch die Gruppierung von Streben wird die Anzahl an Freiheitsgraden reduziert und somit das Optimierungsproblem vereinfacht. Zudem ist die Fertigung von Strukturen welche aus einer beschränkten Anzahl unterschiedlicher Bauteile bestehen deutlich wirtschaftlicher.

Als Freiheitsgrade werden das Strebenprofil pro Gruppe und die Anzahl an Streben pro Gruppe eingeführt. Die Einteilung der Streben zu den Gruppen erfolgt basierend auf der vorliegenden Beanspruchung der jeweiligen Strebe. Die Anzahl an Streben pro Gruppe ist nicht beschränkt, nur die Summe muss der Anzahl an Streben entsprechen:

$$
\sum_{j=1}^{N} n_{j}=I
$$

$N$ ist die Anzahl an Gruppen, $n$ ist die Anzahl an Streben pro Gruppe und $I$ ist die Anzahl an Streben im Fachwerk. Durch Gleichung 5 kann die Anzahl an Streben einer Gruppe bestimmen werden, wodurch sich die Anzahl an Freiheitsgraden $k$ um eins reduziert: 


$$
k=2 N-1 \quad 0<N<I
$$

\subsection{Anfangspopulation}

Die Anfangspopulation wird mit Hilfe des Intervallhalbierungsverfahrens erzeugt. Die Streben werden entsprechend der vorliegenden Beanspruchungen in die Gruppen eingeteilt. Hierfür werden in einem ersten Berechnungsdurchlauf mit gleichem Querschnitt für sämtliche Streben die Beanspruchungen ermittelt. Hieraus kann das Beanspruchungsverhältnis der Streben berechnet werden. Basierend auf dem Beanspruchungsverhältnis $r_{i}$ erfolgt die Ermittlung von $N$ mittleren Beanspruchungsverhältnissen $\bar{r}_{n}$ derart, dass das Minimum der Fehlerquadrate erreicht wird.

$$
\begin{aligned}
& r_{i}=\frac{F_{i}}{F_{\max }} \\
& \min f\left(\bar{r}_{n}\right)=\sum_{i=1}^{I} \min \left(\left(\bar{r}_{1}-r_{i}\right)^{2}, \ldots,\left(\bar{r}_{N}-r_{i}\right)^{2}\right)
\end{aligned}
$$

Die größte auftretende Beanspruchung ist $F_{\text {max }}$. Die Bestimmung des Profils aus dem Baukasten für eine Strebe erfolgt über die Minimierung der Differenz des Beanspruchungsverhältnisses zum Steifigkeitsverhältnis.

$$
\min f\left((E A)_{m}\right)=\left|\bar{r}_{n}-\frac{(E A)_{m}}{(E A)_{\max }}\right|
$$

In Gleichung 9 ist $E$ der Elastizitätsmodul und $A$ die Querschnittsfläche. Durch die Minimierung von Gleichung 9 wird für jedes mittlere Beanspruchungsverhältnis $\bar{r}_{n}$ - somit für jede Strebengruppe - das passende Profil ausgewählt. Somit wird die Annahme getroffen, dass hoch belastete Streben im Allgemeinen einen hohen Beitrag zur Verschiebung der Knoten leisten. Durch die Definition von $(E A)_{\max }$, welches aus der Menge $S$ gewählt wird und die Einhaltung von Gleichung 9 können sämtliche Profile für die Gruppen bestimmt werden. Der einzige verbleibende Freiheitsgrad ist somit $(E A)_{\max }$, welcher mit dem Intervallhalbierungsverfahren ermittelt wird. Das Intervall reicht vom weichsten bis zum steifsten Profil und wird mit jedem Schritt halbiert, wobei die Entscheidung welches verbleibende Teilintervall weiterverwendet wird durch die Nebenbedingung in Gleichung 2 erfolgt. Sobald keine weitere Halbierung des Intervalls mehr möglich ist, ist das Verfahren konvergiert. Die maxi- 
male Anzahl an benötigten Iterationsschritten ist durch die Anzahl an vorhandenen Profilen im Baukasten bestimmt und ist proportional zum Logarithmus der Anzahl an Profilen.

Die gefundene Lösung wird als Teil der Anfangspopulation verwendet. Die restlichen Individuen der Population werden per Zufall erzeugt um die benötigte Vielfalt der Datensätze für den GA zu gewährleisten [11].

\subsection{Optimierungsalgorithmus}

Die Gleichungen 1-3 werden mit dem GA nach $[13,14]$ für diskrete Variablen gelöst. Hierbei werden zuerst Lösungen gefunden welche die Nebenbedingungen 2-3 erfüllen. Anschließend erfolgt die Minimierung der Zielfunktion 1. Die Optimierung ist in Matlab Implementiert.

Die Anfangspopulation wird wie im Absatz 2.2 beschrieben erzeugt. Die Population wird auf Einhaltung von Gleichung 2, 3 und 5 überprüft. Bei denjenigen Individuen welche die Nebenbedingungen erfüllen, wird Gleichung 1 zur Bestimmung der Fitnessfunktion ausgewertet. Die Kreuzung, Selektion und Mutation zum Erzeugen der nächsten Generation erfolgt wie von DEEP in [14] beschrieben. Eine Generation besteht aus 200 Individuen. Die Folgegeneration besteht zu $80 \%$ aus gekreuzten Individuen, $5 \%$ der fittesten Individuen werden in die nächste Generation übernommen und die restlichen $15 \%$ sind mutierte Individuen.

Die Berechnung der Verschiebungen wird über ein FEM-Balkenmodell in ANSYS APDL mit BEAM188 Elementen durchgeführt. Das Element mit quadratischer Ansatzfunktion basiert auf der Balkentheorie nach TIMOSHENKO und ist auch für gedrungene Streben geeignet. Das Fachwerk wird somit biegesteif simuliert, in Anlehnung an die geplante konstruktive Ausführung. Mit zehn Elementen pro Strebe sind die Verschiebungen im Modell konvergiert.

\section{Beispielstruktur 25-Streben Fachwerk}

Für die Demonstration der beschriebenen Methode wird das bereits in mehreren Veröffentlichungen $[7,8,10]$ untersuchte 25-Streben Fachwerk angewendet. Es handelt sich um ein räumliches Fachwerk, siehe Bild 1, welches mit einem Lastfall beaufschlagt wird. Die 25 Streben wurden von den Autoren in acht Gruppen mit gleichen Querschnitten eingeteilt und in den bisherigen Veröffentlichungen mit den Materialparametern von Aluminium untersucht. In der Literatur sind keine Arbeiten mit FVK-Fachwerken gelistet. 
In dieser Arbeit ist die absolute Verschiebung von Knoten 1 und 2 auf $15 \mathrm{~mm}$ beschränkt, bei einem Standard-Lastfall [10], welcher an den Knoten 1, 2, 3 und 6 wie folgt anliegt:

$$
\begin{aligned}
& F_{1}=\left(\begin{array}{lll}
4,45 & 45,5 & -22,25
\end{array}\right)^{T} \mathrm{kN} \\
& F_{2}=\left(\begin{array}{lll}
0 & 45,5 & -22,25
\end{array}\right)^{T} \mathrm{kN}
\end{aligned}
$$

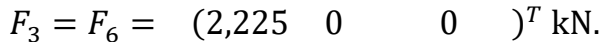

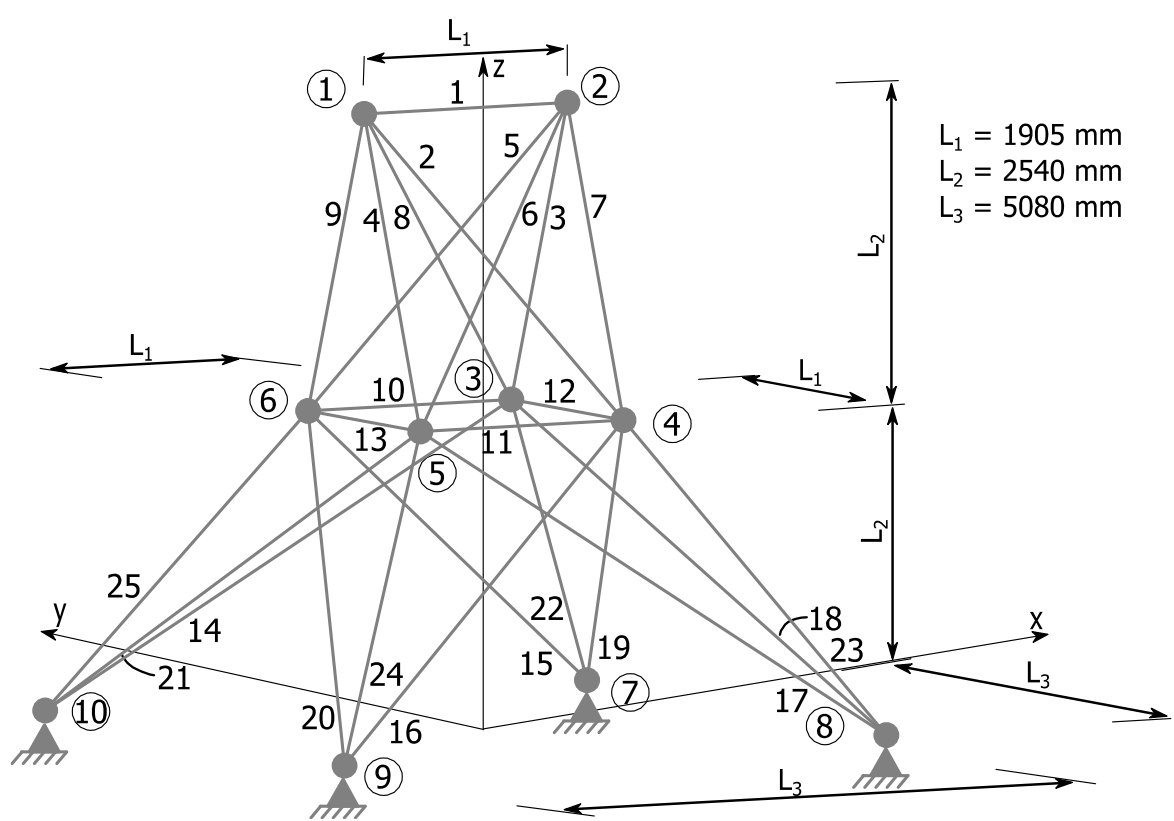

Bild 1: Das 25-Streben Fachwerk. Dargestellt mit Bemaßung und Nummerierung der Streben und Knoten.

Der Baukasten sieht $M=44$ diskrete Profile vor, welche für die einzelnen Streben verwendet werden können. Die Innendurchmesser sind $D_{\text {in }}=\{18 \mathrm{~mm}, 20 \mathrm{~mm}, \ldots, 60 \mathrm{~mm}\}$ wobei jeweils zwei Wandstärken $s_{\mathrm{W}}$ pro Durchmesser angelegt sind $s_{\mathrm{W}}=\{1 \mathrm{~mm}, 2 \mathrm{~mm}\}$ für $D_{\text {in }} \leq 30 \mathrm{~mm}$ und $s_{\mathrm{W}}=\{2 \mathrm{~mm}, 4 \mathrm{~mm}\}$ für $D_{\text {in }}>30 \mathrm{~mm}$. Der Lagenaufbau der Streben ist in Anlehnung an die Untersuchungen von JEGLEY $[15]$ wie folget definiert: $[0, \pm 45,90]$ bei einer prozentualen Verteilung von [80\%,10\%,10\%]. Die Ingenieurskonstanten des Laminates werden, unter Berücksichtigung der Querkontraktionsbehinderung [1], über die Klassische Laminattheorie bestimmt und ergeben sich zu $E_{\mathrm{x}}=151 \mathrm{GPa}$ und $G_{\mathrm{xy}}=11,1 \mathrm{GPa}$. 


\section{Numerisches Beispiel und Ergebnisse}

In diesem Abschnitt werden die Ergebnisse für das in Abschnitt 3 beschriebene Fachwerk präsentiert, wobei folgende Methoden angewendet werden:

- Intervallhalbierungsverfahren,

- GA mit fest definierten Gruppen,

- GA mit variablen Gruppen und zufälliger Anfangspopulation,

- GA mit variablen Gruppen und durch das Intervallhalbierungsverfahren erzeugter Anfangspopulation.

Die Anzahl an Gruppen wurde auf acht festgelegt. Die maximale Durchsenkung auf $u_{\max }=15 \mathrm{~mm}$ beschränkt. Die Anzahl an Streben pro Gruppe und das passende Profil werden durch den Optimierer bestimmt. Da der GA zu den stochastischen Verfahren zählt, wurde die Berechnung jeweils zehn Mal durchgeführt um die Streuung als Maß für die Robustheit und Wiederholbarkeit bestimmen zu können. Für das deterministische Intervallhalbierungsverfahren kann keine Streuung bestimmt werden.

Tabelle 1: Ergebnis des Intervallhalbierungsverfahrens.

\begin{tabular}{|l|l|l|l|l|l|}
\hline $\begin{array}{l}\text { Gruppen- } \\
\text { nummer }\end{array}$ & $\begin{array}{l}\text { Anzahl } \\
\text { Streben }\end{array}$ & Streben-Nummer & $\begin{array}{l}\text { Profil } \\
\text { ID }\end{array}$ & $\begin{array}{c}D_{\text {in }} / s_{W} \\
\text { in mm }\end{array}$ & $\begin{array}{c}E A \\
\text { in } 10^{6} \mathrm{~N}\end{array}$ \\
\hline 1 & 3 & $6,24,25$, & 1 & $18 / 1$ & 9,01 \\
\hline 2 & 7 & $4,5,9,13,15,17$, & 8 & $24 / 2$ & 24,7 \\
\hline 3 & 5 & $1,10,11,18,20$ & 12 & $28 / 2$ & 28,5 \\
\hline 4 & 2 & 2,19 & 15 & $32 / 2$ & 32,3 \\
\hline 5 & 3 & $3,16,14$ & 30 & $46 / 4$ & 94,9 \\
\hline 6 & 2 & 12,7 & 36 & $52 / 4$ & 106 \\
\hline 7 & 2 & 8,23 & 38 & $54 / 4$ & 110 \\
\hline 8 & 1 & 22 & 42 & $58 / 4$ & 118 \\
\hline
\end{tabular}

Das Intervallhalbierungsverfahren ist nach fünf Iterationen konvergiert, wobei sieben Funktionsauswertungen nötig sind. Die Masse der durch das In- 
tervallhalbierungsverfahren gefundenen Lösung beträgt $36,9 \mathrm{~kg}$ bei einer maximalen Verschiebung von Knoten 1 um $u_{1}=14,8 \mathrm{~mm}$. Die gefundene Lösung durch das Intervallhalbierungsverfahren ist in Tabelle 1 im Detail aufgeführt.

In Bild 2 sind die Masse und die Anzahl an benötigten Iterationen für die verwendeten Methoden aufgeführt. Aus dem Diagramm geht hervor, dass mit dem Intervallhalbierungsverfahren das schlechterste Ergebnis bei geringsten Aufwand erzielt wird. Sowohl der Berechnungsaufwand als auch die Masse der gefundenen Lösungen sinken von der ursprünglichen Formulierung des GA mit festen Gruppen hin zum GA mit durch das Intervallhalbierungsverfahren (Bisektion) erzeugter Anfangspopulation. Die Streuung ist für die drei Verfahren vergleichbar und entspricht ebenfalls der Streuung in anderen Arbeiten [10, $16]$.

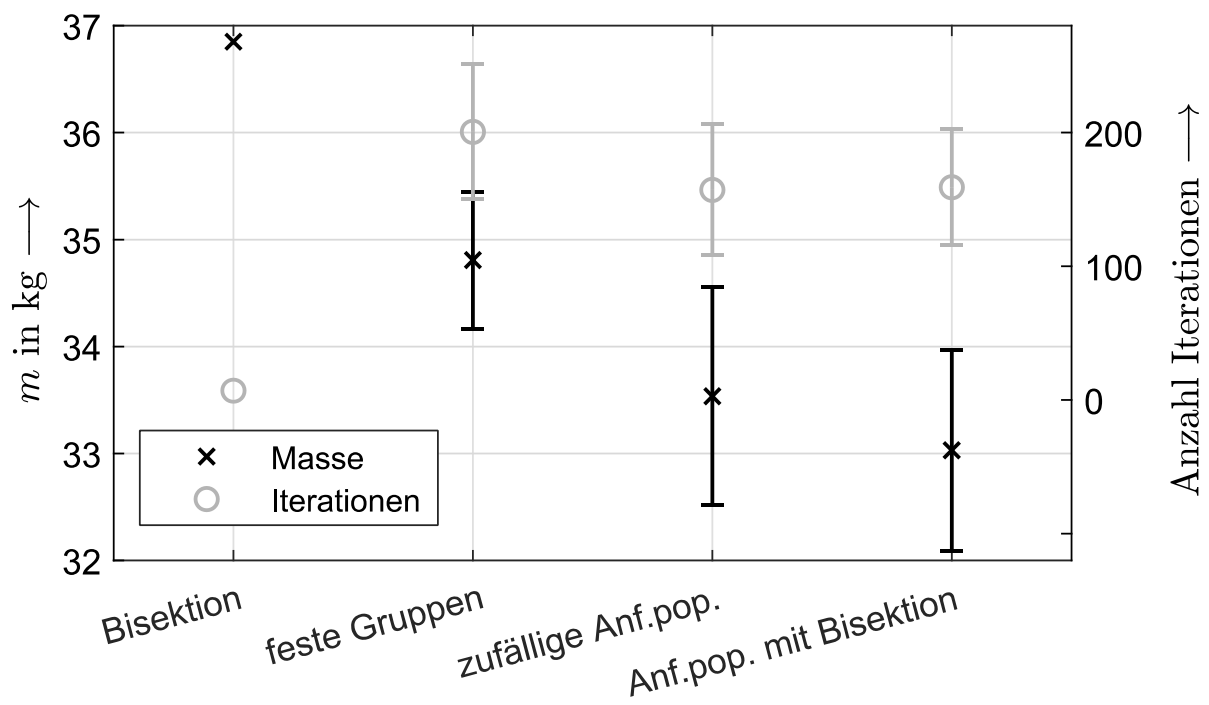

Bild 2: Vergleich der Methoden und Formulierungen.

Die beste gefundene Lösung mit 31,3 kg ist im Vergleich zur Methode mit festen Gruppen mit 34,2 kg um $9 \%$ leichter und benötigt $20 \%$ weniger Iterationen. Die Anzahl an Funktionsauswertungen beträgt jeweils ca. 35000 . Der beste durch den GA gefundene Lösungsvektor ist in Tabelle 2 in Detail aufgeführt. 
Ein quantitativer Vergleich der gefundenen Lösung mit Literaturwerten ist nicht möglich, da bisherige Untersuchungen ausschließlich mit den mechanischen Eigenschaften von Aluminium, Streben mit Kreisquerschnitt und ungebräuchlichen Querschnittswerten [7] durchgeführt wurden.

Bemerkenswert an der gefundenen Lösung ist, dass jeweils zwei Gruppen das gleiche Profil zugeordnet worden ist, wodurch nur noch sechs unterschiedliche Strebenprofile in der Lösung auftauchen.

Tabelle 2: Details der besten Lösung.

\begin{tabular}{|l|l|l|l|l|l|}
\hline $\begin{array}{l}\text { Gruppen- } \\
\text { nummer }\end{array}$ & $\begin{array}{l}\text { Anzahl } \\
\text { Streben }\end{array}$ & Streben-Nummer & $\begin{array}{l}\text { Profil } \\
\text { ID }\end{array}$ & $\begin{array}{c}D_{\text {in }} / s_{W} \\
\text { in mm }\end{array}$ & $\begin{array}{c}E A \\
\text { in } 10^{6} \mathrm{~N}\end{array}$ \\
\hline 1 & 8 & $\begin{array}{l}4,6,9,13,17,21, \\
24,25\end{array}$ & 1 & $18 / 1$ & 9,01 \\
\hline 2 & 1 & 20 & 1 & $18 / 1$ & 9,01 \\
\hline 3 & 6 & $1,5,10,11,15,18$ & 7 & $24 / 1$ & 11,9 \\
\hline 4 & 2 & 2,19 & 19 & $36 / 2$ & 36,1 \\
\hline 5 & 1 & 3 & 21 & $38 / 2$ & 38,0 \\
\hline 6 & 5 & $7,8,12,14,16$ & 34 & $50 / 4$ & 102 \\
\hline 7 & 1 & 23 & 34 & $50 / 4$ & 102 \\
\hline 8 & 1 & 22 & 42 & $58 / 4$ & 117 \\
\hline
\end{tabular}

\section{Diskussion}

Eine neue erweiterte Strategie zur automatischen Gruppierung von Streben in einem Fachwerk wird vorgestellt mit dem Ziel, die Masse zu minimieren bei diskreten Strebenquerschnitten. Damit der Optimierer nicht in einem lokalen Minimum stockt und um die Anzahl an benötigten Generationen zu reduzieren, wird mit dem Intervallhalbierungsverfahren eine Anfangspopulation erzeugt. Die Effektivität und Effizienz des Ansatzes wird an einem Demonstrator gezeigt.

Die numerischen Beispiele legen dar, dass die Optimierung der Gruppengrößen in Kombination mit der Erzeugung einer Anfangspopulation durch das Intervallhalbierungsverfahren eine deutliche Verbesserung des Ergebnisses ermöglichen. Es wird gefolgert, dass das Erzeugen einer deterministischen An- 
fangspopulation im Gegensatz zum Benutzen einer zufällig erzeugten Anfangspopulation für dieses Optimierungsproblem eine Steigerung der Effizienz und Robustheit ermöglicht bei gleichzeitiger Reduktion der nötigen Generationen.

Die dargestellte Formulierung der Optimierung führt trotz Gruppierung der Streben dazu, dass sehr aufwendige und uneinheitliche Knotenkonstruktionen in der Lösung enthalten sind. Eine Lösung dieses Problems könnte durch eine vorgelagerte Ähnlichkeitsbetrachtung der Knoten erfolgen. Die Einteilung der Streben in Gruppen könnte dann neben der vorliegenden Beanspruchung zusätzlich berücksichtigen, dass Knoten mehrfach im Fachwerk auftauchen sollen.

Zum Schluss soll betont werden, dass der vorgeschlagene Algorithmus in der Lage ist, das Optimum zu finden, wobei lediglich die Anzahl an unterschiedlichen Streben-Profilen vorgegeben werden muss, die restlichen Randbedingungen werden vom Algorithmus berücksichtigt. Somit stellt der gefundene Ansatz eine Möglichkeit dar, die Auslegung von Fachwerken auf Basis eines Baukastensystems zu automatisieren.

\section{Danksagung}

Das Projekt wurde gefördert durch das Bundesministerium für Wirtschaft und Energie aufgrund eines Beschlusses des Deutschen Bundestages unter der Fördernummer ZF4222610PO8.

Literatur

[1] Schürmann, H.: "Konstruieren mit Faser-Kunststoff-Verbunden", Springer-Verlag, Berlin, Heidelberg, 2007.

[2] Klein, D.; Witzgall, C.; Wartzack, S.: "A novel approach for the evaluation of composite suitability of lightweight structures at early design stages", International Design Conference - DESIGN, 2014, 1093-1104.

[3] Rathert, T.; Witzgall, C.; Wartzack, S.: "Modular rapid design of multimaterial lightweight truss structures - A novel approach", Book of Abstracts, Symposium Lightweight Design in Product Development, Ermanni, P., et al., Zürich, 2018, 21-23.

[4] Eickenbusch, H.; Krauss, O.: "Kohlenstofffaserverstärkte Kunststoffe im Fahrzeugbau, Ressourceneffizienz und Technologien", VDI Zentrum Ressourceneffizienz, Berlin, 2013. 
[5] Dorn, W.; Gomory, R.; Greenberg, H.: "Automatic design of optimal structures", Journal de Mecanique, 3, 1964, 25-52.

[6] Toakley, A.R.: "Optimum design using available sections", Journal of the Structural Division, 94, 1968, 1219-1244.

[7] Stolpe, M.: "Truss optimization with discrete design variables: a critical review", Structural and Multidisciplinary Optimization, 53, 2016, 349374.

[8] Rajeev, S.; Krishnamoorthy, C. S.: "Discrete Optimization of Structures Using Genetic Algorithms", Journal of Structural Engineering, 118, 1992, 1233-1250.

[9] Wu, S.-J.; Chow, P.-T.: "Steady-state genetic algorithms for discrete optimization of trusses", Computers \& Structures, 56, 1995, 979-991.

[10] Toğan, V.; Daloğlu, A. T.: "An improved genetic algorithm with initial population strategy and self-adaptive member grouping", Computers \& Structures, 86, 2008, 1204-1218.

[11] Schumacher, A.: "Optimierung mechanischer Strukturen, Grundlagen und industrielle Anwendungen", Springer, Berlin, 2005.

[12] Krishnamoorthy, C. S.; Prasanna Venkatesh, P.; Sudarshan, R.: "ObjectOriented Framework for Genetic Algorithms with Application to Space Truss Optimization", Journal of Computing in Civil Engineering, 16, 2002, 66-75.

[13] Deb, K.: "An efficient constraint handling method for genetic algorithms", Computer Methods in Applied Mechanics and Engineering, 186, 2000, 311-338.

[14] Deep, K., et al.: "A real coded genetic algorithm for solving integer and mixed integer optimization problems", Applied Mathematics and Computation, 212, 2009, 505-518.

[15] Jegley, D. C., et al.: "Structural Efficiency of Composite Struts for Aerospace Applications", Journal of Spacecraft and Rockets, 49, 2012, 915924.

[16] Dede, T.; Bekiroğlu, S.; Ayvaz, Y.: "Weight minimization of trusses with genetic algorithm", Applied Soft Computing, 11, 2011, 2565-2575. 